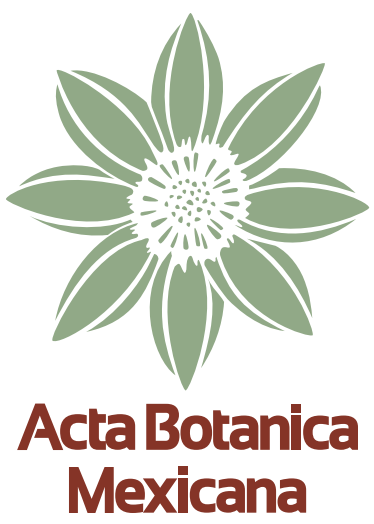

\title{
Caracterización citológica en Flaveria bidentis y $F$. haumanii (Asteraceae)
}

\section{Cytological characterization in Flaveria bidentis and $F$. haumanii (Asteraceae)}

\author{
Valeria de los A. Páez ${ }^{1,4}$ (D), Aldo R. Andradal (D, María T. Sobrero² (D), Salvador Chaila ${ }^{3}$ (D)
}

\section{Resumen:}

Antecedentes y Objetivos: Flaveria es un género americano integrado por 23 especies y representado en Argentina por dos de ellas, ambas nativas y anuales: $F$. bidentis (la cual es considerada maleza de agroecosistemas) y $F$. haumanii. El género tiene un número cromosómico básico de $\mathrm{x}=18$ y los antecedentes citológicos son referidos a $F$. bidentis, pero no así para $F$. haumanii. El objetivo de este trabajo fue caracterizar citogenéticamente a $F$. bidentis y $F$. haumanii a través del análisis cariotípico, el comportamiento meiótico y la estimación de la viabilidad de los granos de polen.

Métodos: El material fue recolectado en la provincia de Santiago del Estero (Argentina) Las raíces fueron pretratadas con 8-hidroxiquinoleina 0.002 M durante ocho horas. Inflorescencias y raíces fueron fijadas en Farmer y conservadas en alcohol etílico al 70\% en frío. Para la tinción de los cromosomas se utilizó hematoxilina propiónica al $2 \%$. La viabilidad de los granos de polen se estimó mediante las soluciones colorantes de Mützing y DAF. Resultados clave: Flaveria bidentis y $F$. haumanii poseen como número cromosómico esporofítico $2 n=36$, con un cariotipo unimodal simétrico. El comportamiento meiótico es regular y en diacinesis se observó $n=18$ bivalentes; la viabilidad estimada de los granos de polen fue superior a $90 \%$.

Conclusiones: Se mencionan por primera vez los números cromosómicos esporofíticos y gametofíticos de $F$. haumanii; además, se completa la información citogenética para F. bidentis a través del análisis de la meiosis. Estos son los primeros datos relacionados con la viabilidad de los granos de polen.

Palabras clave: cariotipo, comportamiento meiótico, mitosis, viabilidad del polen.

\section{Abstract:}

Background and Aims: Flaveria is an American genus with 23 species; in Argentina it is represented by two of them native and annual: F. bidentis (which is cited as a weed of agroecosystems) and $F$. haumanii. The genus is mentioned with a base chromosome number of $\mathrm{x}=18$ and cytogenetic backgrounds for $F$. bidentis are mentioned, but not so for $F$. haumanii. The aim of this work was to characterize $F$. bidentis and $F$. haumanii cytogenetically through the analysis of mitosis, meiotic behavior and estimation of pollen grains viability.

Methods: The material was collected in the province of Santiago del Estero (Argentina). The root tips were pretreated with 8-hydroxyquinoline 0.002 $\mathrm{M}$ for eigth hours. Inflorescences and root tips were fixed in Farmer and preserved in $70 \%$ ethyl alcohol cold. To stain of chromosomes $2 \%$ propionic hematoxylin was used. The viability of pollen grains was estimated with the Mützing and DAF stain solutions.

Key results: Flaveria bidentis and $F$. haumanii have a sporophytic chromosome number of $2 n=36$, with a symmetric unimodal karyotype. The meiotic behavior was regular, in diakinesis $n=18$ bivalent was observed and the estimated viability of the pollen grains was greater than $90 \%$.

Conclusions: The sporophytic and gametophytic chromosomal numbers of $F$. haumanii are mentioned for the first time; in addition, through meiotic analyze the cytogenetic information is completed for $F$. bidentis. These are the first data related to pollen grains viability.

Key words: karyotype, meiotic behavior, mitosis, pollen viability.

${ }^{1}$ Fundación Miguel Lillo, Miguel Lillo 251, 4000 Tucumán, Argentina.

${ }^{2}$ Universidad de Santiago del Estero, Facultad de Agronomía y Agroindustrias, Avenida Belgrano sur 1912, 4200 Santiago del Estero, Argentina.

${ }^{3}$ Universidad Nacional de Tucuman, Facultad de Agronomía y Zootecnia, Av. Roca 1900, 4000 Tucumán, Argentina.

${ }^{4}$ Autor para la correspondencia: paezvaleria@hotmail. com
Recibido: 31 de mayo de 2019. Revisado: 12 de agosto de 2019.

Aceptado por Rosario Redonda Martínez: 23 de septiembre de 2019.

Publicado Primero en línea: octubre de 2019.

Publicado: Acta Botanica Mexicana 127 (2020).
Citar como:

Páez, V. A., R. A. Andrada, M. T. Sobrero y S. Chaila. 2019(2020). Caracterización citológica en Flaveria bidentis y F. haumanii (Asteraceae). Acta Boanica Mexicana 127: e1567. DOI: 10.2189/abm127.2020.1567 


\section{Introducción}

Flaveria Juss. (Subfamilia Asteroideae, Tribu Tageteae) es un género que incluye 23 especies americanas, distribuidas en el Caribe, desde el centro y sur de Estados Unidos de América hasta el norte de Centroamérica y desde Venezuela extendiéndose hasta las regiones del Noroeste, Noreste, Centro y la Patagonia de Argentina (Powell, 1978; Novara y Petenatti, 2012). El género está representado en Argentina por dos especies: Flaveria bidentis (L.) Kuntze y F. haumanii Dimitri \& Orfila (Bártoli et al., 2008). Estos taxones son hierbas nativas, anuales, F. bidentis ocurre de los 0-3000 m s.n.m. y F. haumanii entre 0-1500 m s.n.m. (Petenatti y Ariza Espinar, 1997; Zuloaga y Morrone, 1999; Bártoli et al., 2008; Novara y Petenatii, 2012). A su vez, F. bidentis se ha citado como especie invasora en países de otros continentes, entre ellos Egipto (África), Francia e Inglaterra (Europa), Japón y China (Asia), y Australia (Oceanía) (Chai et al., 2012).

Morfológicamente, Flaveria se caracteriza por la reducción de piezas florales, en muchas especies, las diferencias se basan en la distribución que presentan, hábitat, follaje, capitulescencias las que pueden estar formadas por cabezas fuertemente agrupadas o laxas, en panículas corimbosas generalmente planas, o inflorescencias similares a glomérulos axilares sésiles; cabezuelas radiadas o discoides (Powell, 1978).

Una característica de las plantas anuales, como Flaveria bidentis y $F$. haumanii, es que tienen sólo una oportunidad durante su ciclo de vida para reproducirse y dejar descendencia, debido a ello, la supervivencia de estas especies depende de las condiciones ambientales prevalecientes, motivo por el cual la autofertilización puede considerarse un modo de reproducción sexual muy ventajoso (Proctor y Yeo, 1979). En las plantas donde las visitas de los polinizadores son limitadas y tienen estrategia monocárpica; la autogamia es un mecanismo que asegura la descendencia (Hagerup, 1951; Baker, 1955; Stebbins, 1957; Richards, 1986). El número y tamaño de los cromosomas tiene incidencia directa en los valores del índice de recombinación/ número de entrecruzamientos que tienen lugar durante la meiosis I (Darlington, 1939), los cuales están estrechamente relacionados con el modo de fertilización de las plantas; las especies con autofecundación se caracterizan por pre- sentar complementos cromosómicos dotados de pocos y pequeños cromosomas, es decir, factores que generan la reducción potencial de quiasmas. Esta característica condiciona los sistemas genéticos con directa incidencia sobre el valor de recombinación total, afectando la variabilidad natural (Stebbins, 1971; Gabriele et al., 2009).

Citológicamente, en Asteraceae, solo una pequeña parte de las especies han sido analizadas y los recuentos de sus cromosomas han determinado una gran variedad en los números cromosómicos, desde $n=2$, por ejemplo, en Xanthisma gracile (Nutt.) D.R. Morgan \& R.L. Hartm. y Brachyscome lineariloba (DC.) Druce, hasta formas poliploides con $n=432$ aproximadamente, en Olearia albida Hook $f$. (Beuzenberg y Hair, 1984).

Powell (1978) mencionó como número cromosómico más representativo para Flaveria $\mathrm{n}=18$, esto se ve reflejado en 18 de las 21 especies que cuentan con recuentos cromosómicos (Keil y Stuessy, 1977; Powell, 1978; Powell y Powell, 1978). Existen conteos cromosómicos gametofíticos de $\mathrm{n}=18$ para $F$. bidentis provenientes de material de la flora Argentina y un recuento esporofítico de $2 n=36$ perteneciente a ejemplares de la flora de China (Powell y Powell, 1978; Robinson et al., 1981; Hunziker et al., 1990; Shi Li et al., 2006). Por el contrario, hasta el momento F. haumanii no cuenta con recuentos. El objetivo de este trabajo fue caracterizar citogenéticamente a $F$. bidentis y $F$. haumanii a través del análisis de la mitosis, el comportamiento meiótico y la estimación de la viabilidad del polen.

\section{Materiales y Métodos}

\section{Material estudiado}

El material fue recolectado en la provincia de Santiago del Estero (Argentina) y depositado en el Herbario Fanerogámico de la Fundación Miguel Lillo (LIL).

Material examinado de Flaveria bidentis: ARGENTINA. Provincia Santiago del Estero, Departamento San Pedro, Zanjón, 178 m s.n.m., 27ํ51'05.89"S, 6414'34.19"O, 09.V.2014, V. de los A. Páez s.n. (LIL 613.740 A, B).

Material examinado de Flaveria haumanii: ARGENTINA. Provincia Santiago del Estero, Departamento Ojo de Agua, Isla Verde, 125 m s.n.m., 28³8'27.87"S, 6405'12.35"O, 09.V.2014, V. de los A. Páez s.n. (LIL 613.733 $A, B, C)$. 


\section{Metodología}

\section{Mitosis}

Para el estudio se obtuvieron radículas de semillas germinadas que se pretrataron con 8-hidroxiquinoleina $0.002 \mathrm{M}$ durante ocho horas a temperatura ambiente, posteriormente se fijaron en Farmer (alcohol etílico-ácido acético, $3: 1)$ por 24 horas, y fueron conservadas a $4{ }^{\circ} \mathrm{C}$ en alcohol etílico $70 \%$. Las raíces se hidrolizaron en $\mathrm{HCl} 1 \mathrm{~N}$ durante 15 minutos a $60{ }^{\circ} \mathrm{C}$ (Estufa Dalvo MCM2, Industria Argentina), con posterior lavado en agua destilada, después se colorearon y montaron con una gota de hematoxilina propiónica al $2 \%$.

Los parámetros cariotípicos se obtuvieron a partir de siete placas metafásicas y las mediciones se realizaron con el programa Micromeasure 3.3 (Reeves, 2001). Se analizó, para cada par cromosómico, la longitud total de los cromosomas (c); longitud del brazo largo (I) y corto (s), longitud total del complemento cromosómico haploide (TCL) y, para el conjunto cromosómico de cada célula, la longitud cromosómica media (C), longitud cromosómica máxima y mínima (C máxima, C mínima) e índice centromérico (Ic). Los cromosomas se clasificaron de acuerdo a la metodología propuesta por Levan et al. (1964) y las asimetrías cromosómicas se calcularon de acuerdo a la metodología propuesta por Romero-Zarco (1986).

\section{Análisis estadístico}

Para estudiar la relación entre las variables (c, l, s, Ic) de las especies, se llevó a cabo un experimento factorial 2 (especies) $\times 18$ (pares de cromosomas) en un diseño completamente aleatorizado. Para el análisis se emplearon modelos lineales generales y mixtos, considerando a la especie, al par de cromosomas y a su interacción como factores fijos y se modeló la variancia con diferentes funciones. Con el propósito de determinar diferencias significativas entre las medias de las variables de las especies, se utilizó la prueba $\mathrm{Di}$ Rienzo, Guzmán y Casnoves o DGC (Di Rienzo et al., 2012). Con la finalidad de modelar la varianza se utilizaron los criterios de Akaike AIC (Akaike, 1973) y Bayesiano de Schwarz BIC (Schwarz, 1978), seleccionándose el modelo que tuvo el menor valor de AIC y BIC. Los datos se analizaron mediante el empleo del programa InfoStat (Di Rienzo et al., 2012).

\section{Meiosis}

Se recolectaron botones florales que se fijaron, colorearon y conservaron con el mismo protocolo empleado para el tratamiento de los meristemas radiculares. Se examinaron 50 células por cada estadio de la meiosispara este análisis. En diacinesis se estimó el número de bivalentes, la frecuencia de quiasmas e índice de recombinación de cada especie analizada. Se calculó el índice de recombinación (RI) de ambos taxones, el cual es una medida de la recombinación meiótica total intra e intercromosómica, que se obtuvo mediante la suma del número haploide de cromosomas ( $\mathrm{n}$ ) y el promedio de quiasmas por célula (Q/II) (Darlington, 1939).

\section{Viabilidad de polen}

La estimación de la viabilidad de los granos de polen se llevó a cabo con dos técnicas: a) la solución de Mützing (glicerina-carmín 1:1) para material fijado (Sharma y Sharma, 1965) y b) el fluorocromo diacetato de fluoresceína (DAF), en flores inmediatamente después de la antesis (HeslopHarrison y Heslop-Harrison, 1970). En ambos casos se tomaron diez flores al azar, y como valor mínimo 1000 granos de polen.

Las microfotografías se obtuvieron con una cámara Qcolor-5 (Ontario, Canadá) montada a un microscopio Olympus BX43 (Tokio, Japón). Los idiogramas se realizaron con el software CorelDrawX3.

\section{Resultados}

\section{Mitosis}

El estudio mitótico evidenció que ambas especies poseen un número cromosómico esporofítico $2 n=36$ (Figs. 1A-B). La fórmula cariotípica registrada para F. bidentis y $F$. haumanii fue de $36 \mathrm{~m}$. En el Cuadro 1 se registran los parámetros morfométricos del complemento cromosómico para cada especie analizada.

En Flaveria bidentis la longitud total del complemento cromosómico y la longitud cromosómica media registrada fueron de 68.98 y $1.91 \mu \mathrm{m}$ respectivamente (Cuadro 2); la variación de sus longitudes cromosómicas medias osciló entre 3.08-1.35 $\mu \mathrm{m}$ (Cuadro 1). El índice centromérico medio tuvo un valor de 42.15 colocando a sus cromosomas en la categoría de metacéntricos (Fig. 1C). El cariotipo es unimodal por la semejanza en el tamaño cromosómico, justifi- 


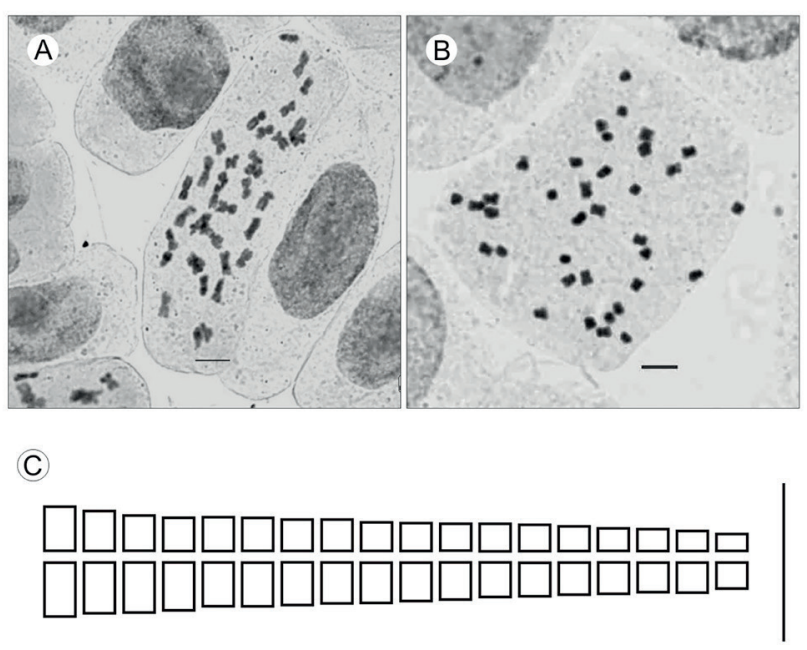

(D)

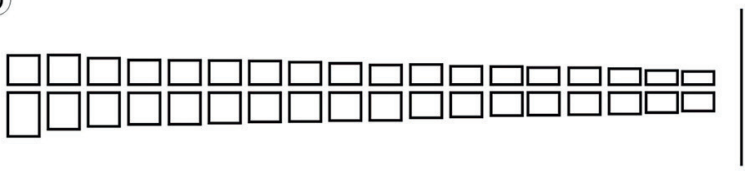

Figura 1: Cariotipo e idiograma de Flaveria bidentis (L.) Kuntze y $F$. haumanii Dimitri \& Orfila. A, C. Flaveria bidentis. B, D. Flaveria haumanii. Escala $=5 \mu \mathrm{m}$.

cado por el valor del índice de asimetría intercromosómica $A_{2}=0.20$. El índice de asimetría intracromosómica indica un cariotipo simétrico con $\mathrm{A}_{1}=0.30$ (Cuadro 2).

En Flaveria haumanii, el complemento cromosómico total y la longitud cromosómica media calculados fueron de 54.65 y $1.51 \mu \mathrm{m}$ respectivamente (Cuadro 2), mientras que las longitudes cromosómicas medias variaron entre 2.48$0.98 \mu \mathrm{m}$ (Cuadro 1). El valor del índice centromérico medio de 43.07 refleja cromosomas con categoría de metacéntrico (Fig. 1D). El cariotipo es unimodal y simétrico con valores de $A_{2}=0.22$ y $A_{1}=0.32$ respectivamente (Cuadro 2 ).

\section{Análisis estadístico}

El análisis estadístico, mostró que los factores (especies) resultaron significativos, no así la interacción ( $p=0.99)$, por lo que los factores "especie y par de cromosomas" actúan en forma independiente. Para las variables analizadas al comparar las medias con la prueba DGC, mostró menores valores en F. haumanii (1.55) con respecto a F. bidentis (2.16). El análisis del índice centromérico, no resultó significativo para las variables especie, par de cromosomas e interacción.
El análisis estadístico de la variable c para ambas especies mostró diferencias significativas formándose tres grupos, el primero comprende los pares cromosómicos 1-8, el segundo con los pares 9-13 y el tercero incluye los pares 14-18. Para la variable I, el análisis determinó tres grupos que no se corresponden con los anteriores, un grupo comprende los pares 1-3, otro abarca los pares 4-10, y el tercero, del par 11-18. Finalmente, la variable s arrojó cuatro grupos: el primero abarca los pares 1-2, el segundo los pares 3-9, el tercero los pares $10-16$ y el último incluye los pares 17-18 (Cuadro 3).

\section{Meiosis}

En el análisis de la meiosis de las dos especies estudiadas, se observó como número gametofítico $n=18$ (Figs. 2A-B). En F. bidentis las células madre del polen (CMP) durante diacinesis presentaron la mayoría de sus bivalentes cerrados (54.61\%) con quiasmas distales (70.66\%), el promedio de quiasmas por bivalente $(\mathrm{Q} / \mathrm{II})$ fue de 1.54 y el índice de recombinación $\mathrm{IR}=45.82$; mientras que en $F$. haumanii los bivalentes son cerrados $(38.88 \%)$ con quiasmas distales (56.65\%), el promedio de quiasmas por bivalente es de 1.37 y el IR=42.71 (Cuadro 4).

En la primera etapa de la división meiótica se observaron en F. bidentis metafase I (MI) con $8 \%$ de sus cromosomas fuera de placa ecuatorial, desde la anafase (Al) hasta la telofase (TI) las células exhibieron un comportamiento meiótico regular. En la segunda división meiótica se presentaron células regulares en los diferentes estadios; asimismo, durante el desarrollo de las tétradas no se observaron irregularidades.

En F. haumanii, en ambas etapas de la división, se observaron células con irregularidades meióticas con baja frecuencia, sólo $2 \%$ de las CMP con cromosomas fuera de la placa ecuatorial. El comportamiento de los cromosomas durante la división meiótica fue normal y la formación de tétradas regular.

\section{Viabilidad de Polen}

La estimación de la viabilidad de los granos de polen determinada con la solución de Mützing fue de $95.20 \%$ en $F$. bidentis y $90 \%$ en $F$.haumanii (Fig. $2 \mathrm{C}$ ), mientras que con DAF en $F$. bidentis arrojó valores de $90 \%$ (Fig. 2D) y en $F$. 
Cuadro 1: Parámetros morfométricos de los cromosomas de Flaveria bidentis (L.) Kuntze y F. haumanii Dimitri \& Orfila. Referencias: longitud cromosómica total (c), longitud del brazo largo (I), longitud del brazo corto (s), índice centromérico medio (Ic), metacéntrico (m), error estándar (ES).

\begin{tabular}{|c|c|c|c|c|c|}
\hline Par cromosómico & $c(\mu \mathrm{m}) \pm \mathrm{ES}$ & $\mathrm{I}(\mu \mathrm{m}) \pm \mathrm{ES}$ & $s(\mu \mathrm{m}) \pm \mathrm{ES}$ & Ic & Tipo cromosómico \\
\hline \multicolumn{6}{|c|}{ F. bidentis (L.) Kuntze } \\
\hline 1 & $3.08 \pm 0.30$ & $1.70 \pm 0.17$ & $1.38 \pm 0.13$ & 44.8 & $\mathrm{~m}$ \\
\hline 2 & $2.86 \pm 0.28$ & $1.60 \pm 0.17$ & $1.26 \pm 0.13$ & 4.05 & $\mathrm{~m}$ \\
\hline 3 & $2.69 \pm 0.26$ & $1.58 \pm 0.16$ & $1.11 \pm 0.11$ & 41.26 & $\mathrm{~m}$ \\
\hline 4 & $2.55 \pm 0.25$ & $1.50 \pm 0.16$ & $1.05 \pm 0.11$ & 41.17 & $\mathrm{~m}$ \\
\hline 5 & $2.45 \pm 0.23$ & $1.38 \pm 0.13$ & $1.07 \pm 0.11$ & 43.67 & $\mathrm{~m}$ \\
\hline 6 & $2.42 \pm 0.23$ & $1.38 \pm 0.11$ & $1.04 \pm 0.14$ & 42.97 & $\mathrm{~m}$ \\
\hline 7 & $2.34 \pm 0.23$ & $1.35 \pm 0.15$ & $0.99 \pm 0.10$ & 42.3 & $\mathrm{~m}$ \\
\hline 8 & $2.28 \pm 0.22$ & $1.29 \pm 0.12$ & $0.99 \pm 0.10$ & 43.42 & $\mathrm{~m}$ \\
\hline 9 & $2.17 \pm 0.20$ & $1.27 \pm 0.10$ & $0.90 \pm 0.11$ & 41.47 & $\mathrm{~m}$ \\
\hline 10 & $2.09 \pm 0.18$ & $1.21 \pm 0.11$ & $0.88 \pm 0.08$ & 42.1 & $\mathrm{~m}$ \\
\hline 11 & $2.02 \pm 0.18$ & $1.17 \pm 0.12$ & $0.85 \pm 0.09$ & 42.07 & $\mathrm{~m}$ \\
\hline 12 & $1.96 \pm 0.16$ & $1.09 \pm 0.12$ & $0.87 \pm 0.08$ & 44.38 & $\mathrm{~m}$ \\
\hline 13 & $1.89 \pm 0.16$ & $1.06 \pm 0.11$ & $0.83 \pm 0.06$ & 43.91 & $\mathrm{~m}$ \\
\hline 14 & $1.79 \pm 0.16$ & $1.01 \pm 0.09$ & $0.78 \pm 0.08$ & 43.57 & $\mathrm{~m}$ \\
\hline 15 & $1.70 \pm 0.15$ & $0.99 \pm 0.09$ & $0.71 \pm 0.07$ & 41.76 & $\mathrm{~m}$ \\
\hline 16 & $1.62 \pm 0.15$ & $0.93 \pm 0.08$ & $0.69 \pm 0.08$ & 42.59 & $\mathrm{~m}$ \\
\hline 17 & $1.58 \pm 0.14$ & $0.95 \pm 0.06$ & $0.63 \pm 0.09$ & 39.87 & $\mathrm{~m}$ \\
\hline 18 & $1.35 \pm 0.13$ & $0.82 \pm 0.10$ & $0.53 \pm 0.06$ & 39.25 & $\mathrm{~m}$ \\
\hline \multicolumn{6}{|c|}{ F. haumanii Dimitri \& Orfila } \\
\hline 1 & $2.32 \pm 0.13$ & $1.39 \pm 0.11$ & $0.93 \pm 0.05$ & 40.08 & $\mathrm{~m}$ \\
\hline 2 & $2.10 \pm 0.09$ & $1.16 \pm 0.06$ & $0.94 \pm 0.04$ & 44.76 & $\mathrm{~m}$ \\
\hline 3 & $1.91 \pm 0.04$ & $1.07 \pm 0.04$ & $0.84 \pm 0.03$ & 43.97 & $\mathrm{~m}$ \\
\hline 4 & $1.83 \pm 0.04$ & $1.04 \pm 0.04$ & $0.79 \pm 0.03$ & 43.16 & $\mathrm{~m}$ \\
\hline 5 & $1.76 \pm 0.04$ & $1.03 \pm 0.04$ & $0.73 \pm 0.06$ & 41.47 & $\mathrm{~m}$ \\
\hline 6 & $1.73 \pm 0.03$ & $0.97 \pm 0.04$ & $0.76 \pm 0.05$ & 43.93 & $\mathrm{~m}$ \\
\hline 7 & $1.68 \pm 0.02$ & $0.93 \pm 0.02$ & $0.75 \pm 0.03$ & 44.64 & $\mathrm{~m}$ \\
\hline 8 & $1.63 \pm 0.3$ & $0.92 \pm 0.02$ & $0.71 \pm 0.03$ & 43.56 & $\mathrm{~m}$ \\
\hline 9 & $1.56 \pm 0.04$ & $0.89 \pm 0.04$ & $0.67 \pm 0.03$ & 42.95 & $\mathrm{~m}$ \\
\hline 10 & $1.51 \pm 0.04$ & $0.88 \pm 0.04$ & $0.63 \pm 0.03$ & 41.72 & $\mathrm{~m}$ \\
\hline 11 & $1.44 \pm 0.04$ & $0.80 \pm 0.03$ & $0.64 \pm 0.04$ & 44.44 & $\mathrm{~m}$ \\
\hline 12 & $1.35 \pm 0.04$ & $0.77 \pm 0.04$ & $0.58 \pm 0.03$ & 42.96 & $\mathrm{~m}$ \\
\hline 13 & $1.30 \pm 0.05$ & $0.73 \pm 0.05$ & $0.57 \pm 0.02$ & 43.84 & $\mathrm{~m}$ \\
\hline 14 & $1.26 \pm 0.05$ & $0.72 \pm 0.02$ & $0.54 \pm 0.03$ & 42.86 & $\mathrm{~m}$ \\
\hline 15 & $1.24 \pm 0.04$ & $0.70 \pm 0.04$ & $0.54 \pm 0.03$ & 43.55 & $\mathrm{~m}$ \\
\hline 16 & $1.19 \pm 0.05$ & $0.68 \pm 0.04$ & $0.51 \pm 0.02$ & 42.86 & $\mathrm{~m}$ \\
\hline 17 & $1.09 \pm 0.04$ & $0.64 \pm 0.03$ & $0.45 \pm 0.03$ & 41.28 & $\mathrm{~m}$ \\
\hline 18 & $1.00 \pm 0.05$ & $0.58 \pm 0.02$ & $0.42 \pm 0.03$ & 42 & $\mathrm{~m}$ \\
\hline
\end{tabular}


Cuadro 2: Parámetros cariotípicos de Flaveria bidentis (L.) Kuntze y $F$. haumanii Dimitri \& Orfila. Referencias: longitud total del complemento cromosómico haploide (TCL), longitud cromosómica media (C), longitud cromosómica máxima y mínima (C máxima, C mínima), índice centromérico medio (Ic), índice de asimetría intracromósomica (A1), índice de asimetría intercromosómica (A2), metacéntrico (m).

\begin{tabular}{lcc}
\hline Parámetros & $\begin{array}{c}\text { F. bidentis (L.) } \\
\text { Kuntze }\end{array}$ & $\begin{array}{c}\text { F. haumanii Dimitri \& } \\
\text { Orfila }\end{array}$ \\
\hline $2 \mathrm{n}$ & 36 & 36 \\
Formula cariotípica & $36 \mathrm{~m}$ & $36 \mathrm{~m}$ \\
TCL $(\mu \mathrm{m})$ & 68.98 & 54.65 \\
C $(\mu \mathrm{m})$ & 1.91 & 1.51 \\
C máxima $(\mu \mathrm{m})$ & 3.08 & 2.48 \\
C mínima $(\mu \mathrm{m})$ & 1.35 & 0.98 \\
IC & 42.15 & 43.07 \\
A1 & 0.30 & 0.32 \\
A2 & 0.20 & 0.22 \\
\hline
\end{tabular}

haumanii de 74\%. En el Cuadro 4 se resumen los parámetros meióticos analizados en diacinesis, Ml y la viabilidad de polen de F. bidentis y F. haumanii.

\section{Discusión}

Lewis (1980) propuso el número básico x=9 para la familia Asteraceae, y sugirió, que según la tribu analizada pueden presentarse otros dos números básicos diferentes al establecido para la familia: $\mathrm{x}=10$ o 17. Por otro lado, Powell (1978) sostiene que Flaveria presenta un número básico $x=18$, siendo la mayoría de sus especies diploides. No obstante, hay indicios que sugieren un número básico menor a éste. Anderson (1972) observó $\mathrm{n}=9$ para $F$. campestris J.R. Johnst. como ejemplo de polihaploide (planta haploide que deriva de un individuo poliploide). La poliploidía no es común en el género, por lo que Powell (1978) reportó $\mathrm{n}=36$ para F. pringlei Gand., como única especie poliploide encontrada hasta el momento. Los antecedentes citogenéticos revelaron para Flaveria el número básico $\mathrm{x}=18$, por

Cuadro 3: Medias ajustadas para la variable pares cromosómicos por análisis DGC. Medias con una letra común no son significativamente diferentes ( $p>0.05)$. Referencias: longitud cromosómica total (c), longitud del brazo largo (l), longitud del brazo corto (s).

\begin{tabular}{ccccccc}
\hline Par cromosómico & c & I & S & C & I & s \\
\hline 1 & 2.7 & 1.55 & 1.16 & A & D & G \\
2 & 2.48 & 1.38 & 1.10 & A & D & G \\
3 & 2.31 & 1.33 & 0.97 & A & D & H \\
4 & 2.19 & 1.27 & 0.92 & A & E & H \\
5 & 2.11 & 1.21 & 0.90 & A & E & H \\
6 & 2.08 & 1.18 & 0.90 & $\mathrm{~A}$ & $\mathrm{E}$ & $\mathrm{H}$ \\
7 & 2.01 & 1.14 & 0.87 & $\mathrm{~A}$ & $\mathrm{E}$ & $\mathrm{H}$ \\
8 & 1.96 & 1.11 & 0.84 & $\mathrm{~A}$ & $\mathrm{E}$ & $\mathrm{H}$ \\
9 & 1.87 & 1.07 & 0.79 & $\mathrm{~B}$ & $\mathrm{E}$ & $\mathrm{H}$ \\
10 & 1.80 & 1.05 & 0.75 & $\mathrm{~B}$ & $\mathrm{E}$ & $\mathrm{I}$ \\
11 & 1.73 & 0.99 & 0.74 & $\mathrm{~B}$ & $\mathrm{~F}$ & $\mathrm{I}$ \\
12 & 1.66 & 0.93 & 0.73 & $\mathrm{~B}$ & $\mathrm{~F}$ & $\mathrm{I}$ \\
13 & 1.60 & 0.90 & 0.70 & $\mathrm{~B}$ & $\mathrm{~F}$ & $\mathrm{I}$ \\
14 & 1.52 & 0.87 & 0.66 & $\mathrm{C}$ & $\mathrm{F}$ & $\mathrm{I}$ \\
15 & 1.47 & 0.84 & 0.63 & $\mathrm{C}$ & $\mathrm{F}$ & $\mathrm{I}$ \\
16 & 1.41 & 0.81 & 0.6 & $\mathrm{C}$ & $\mathrm{F}$ & $\mathrm{I}$ \\
17 & 1.30 & 0.75 & 0.54 & $\mathrm{C}$ & $\mathrm{F}$ & $\mathrm{J}$ \\
18 & 1.23 & 0.75 & 0.48 & $\mathrm{C}$ & $\mathrm{F}$ & $\mathrm{J}$ \\
\hline
\end{tabular}



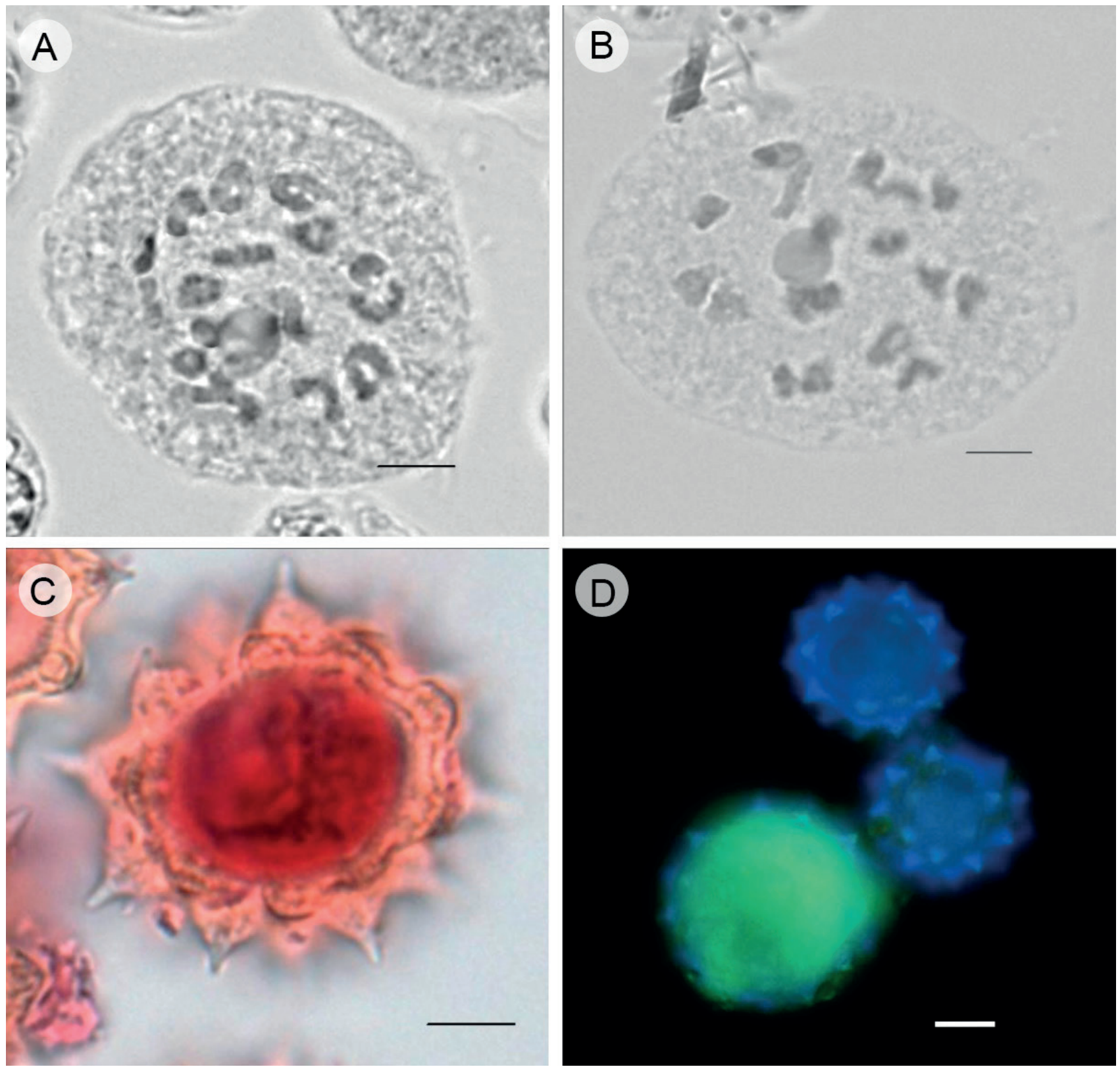

Figura 2: Diacinesis y viabilidad polínica de Flaveria bidentis (L.) Kuntze y F. haumanii Dimitri \& Orfila. A. Flaveria bidentis, diacinesis n=18; B. Flaveria haumanii, diacinesis n=18; C. Flaveria haumanii, grano de polen viable teñido con Mützing; D. Flaveria bidentis, grano de polen viable (fluorescente) y granos inviables teñidos con DAF. Escala $=5 \mu \mathrm{m}$.

lo tanto, los resultados del presente estudio se corresponden con la información mencionada (Turner, 1971, 1975; Powell, 1978), e indican que los taxones analizados son diploides y de comportamiento meiótico regular.

El análisis estadístico en $F$. bidentis y $F$. haumanii corrobora la diferencias entre ellas y esto puede ser observado en los pares cromosómicos antes mencionados para c, I y s. Shi Li et al. (2006) realizaron estudios citogenéticos en una población de $F$. bidentis proveniente de China y regis- traron la fórmula cariotípica de $24 m+8 s m+4 s t$, la longitud total cromosómica de $81.16 \mu \mathrm{m}$ y el rango de variación en los cromosomas de 1.12-3.36 $\mu \mathrm{m}$. Aunque la fórmula cariotípica es disímil a la encontrada en este estudio (36 m), así como también la longitud total cromosómica, el rango de variación se asemeja a lo reportado por estos autores, la causa de esta diferencia podría deberse a alteraciones cromosómicas estructurales, o bien al tiempo de exposición de las raíces al pretratamiento que difiere en ambos estudios. 
Cuadro 4: Síntesis de parámetros meióticos de Flaveria bidentis (L.) Kuntze y F. haumanii Dimitri \& Orfila.

\begin{tabular}{lcc}
\hline Especie & $\begin{array}{c}\text { F. bidentis (L.) } \\
\text { Kuntze }\end{array}$ & $\begin{array}{c}\text { F. haumani Dimitri } \\
\text { \& Orfila }\end{array}$ \\
\hline $\mathrm{n}$ & 18 & 18 \\
$\begin{array}{l}\text { Bivalentes por célula } \\
\pm \mathrm{ES}\end{array}$ & & \\
Cerrados & $9.83 \pm 4.88$ & $7.00 \pm 2.52$ \\
Abiertos & $8.17 \pm 4.88$ & $10.71 \pm 2.50$ \\
Totales & 18 & 18 \\
Quiasma por célula & 93 & 98 \\
\pm ES & $19.67 \pm 9.75$ & $14 \pm 5.03$ \\
Distales & $8.17 \pm 4.88$ & $10.71 \pm 2.5$ \\
Intersticiales & $27.83 \pm 4.88$ & $24.71 \pm 2.63$ \\
Totales & $1.54 \pm 0.27$ & $1.37 \pm 0.15$ \\
Quiasmas por & & \\
bivalente \pm ES & 45.82 & 42.71 \\
Índice de & & \\
Recombinación (IR) & $90 \%$ & \\
Viabilidad de polen & & \\
Mützing & & \\
DAF & & \\
\hline
\end{tabular}

Powell (1978) cita que las especies anuales, entre ellas $F$. bidentis, son autocompatibles y ocasionalmente la autofertilización puede ser estimulada por la fricción de los glomérulos; también mencionó que la fecundación cruzada que exhiben estas especies se debe a insectos polinizadores que se ven atraídos por las flores liguladas. La autofertilización reduce la variabilidad genética dentro de las poblaciones, no obstante, siempre existe cierta cantidad de entrecruzamiento durante las divisiones meióticas que evita o anula por completo la depresión por endogamia (Richards, 1986; Charlesworth y Charlesworth, 1987).

Koella (1993) realizó un análisis de las correlaciones ecológicas entre la frecuencia de quiasmas y el índice de recombinación de diferentes especies de plantas. En la mayoría de las especies con semillas pequeñas y amplia capacidad de dispersión estudiadas por este autor, los valores de IR oscilaron entre 19.4 y 27.1. Estos datos no son acordes a los resultados del presente estudio, debido a que tanto F. bidentis como $F$. haumanii son especies con semillas pequeñas, sin embargo, los valores de IR, son considerablemente mayores a los descriptos por Koella (1993).
Kulshreshtha y Gupta (1979) citan datos referentes a índice de recombinación y frecuencia de quiasma para el género Helianthus L., de un total de 28 taxones analizados, diez son especies anuales diploides con $n=17$; el índice de recombinación de estas especies varía de $22.60-28.83$ y la frecuencia de quiasma entre 1.32-1.57, valores distintos a los encontrados en las especies estudiadas en el presente trabajo. Asimismo, Gabriele et al. (2009) realizaron sus propias investigaciones en Commelina benghalensis L., otra maleza ampliamente distribuida en todos los continentes, los resultados que obtuvieron son disimiles a los de nuestro aporte (IR=37.3 y $Q / I I=1.9)$. Las diferencias pueden deberse a que el IR está directamente influenciado por el tamaño de los cromosomas y el nivel de ploidía de cada especie, presentando valores más grandes cuanto mayor sea el número y tamaño de los cromosomas (Kulshreshtha y Gupta, 1979; Gabriele et al., 2009).

La viabilidad del polen representa una medida confiable de estimación del potencial reproductivo masculino de las plantas (Alexander, 1980), ésta proporciona información necesaria para conocer la capacidad reproductiva de una especie y, con certeza científica, saber las posibilidades de perpetuidad del genoma analizado; además, en la agricultura su importancia se estima como un parámetro válido durante el período de planificación de un cultivo y/o su mejoramiento (Alexander, 1980). Tanto F. bidentis como F. haumanii mostraron alta viabilidad de los granos de polen con las técnicas empleadas, como consecuencia directa del comportamiento meiótico regular, hecho que concuerda con la amplia capacidad reproductiva de ambas especies.

\section{Conclusiones}

Por primera vez se dan a conocer los números cromosómicos gametofítico y esporofítico, para F. haumanii, como así el comportamiento meiótico (incluyendo su capacidad de recombinación) y la viabilidad de los granos de polen para $F$. bidentis y $F$. haumanii. Los datos recabados aquí podrían tener relevancia durante la planificación de estrategias de control, debido a la capacidad invasora de estas plantas.

\section{Contribución de autores}

VAP, MTS y SC diseñaron la presente investigación y colectaron el material. VAP y ARA aplicaron las técnicas citoló- 
gicas convencionales y se encargaron de la adquisición de datos y su interpretación. Todos los autores contribuyeron a la discusión, revisión y aprobación del manuscrito final.

\section{Financiamiento}

Este estudio fue apoyado por la Fundación Miguel Lillo en el marco del proyecto CUP-B-0013-1 (Miguel Lillo 251, T4000JFE, Tucumán) y por el Consejo Investigaciones de Ciencia y Tecnología-Universidad Nacional de Santiago del Estero (CICYT-UNSE), proyecto: Bio-ecología y manejo de malezas de los agroecosistemas del semiárido 23/A199.

\section{Agradecimientos}

Se agradecen las facilidades brindadas por el Instituto Tecnológico de Chiná, Campeche, México. A Pilar Yuriko Cruz Koizumi, Perla Ortíz Colín, Janeth Chan Morales, Jhoanny del C. Poot Castro, Geovanny J. Canul Hernández, Edwin A. Poot Euan y Carlos F. Vera Mendicuti por el apoyo brindado durante la realización del experimento.

\section{Literatura citada}

Akaike, H. 1973. Information theory and an extension of the maximum likelihood principle. In: Petrov, B. N. y F. Csaki (eds.). Second International Symposium on Information Theory. Akademiai Kiado. Budapest, Hungary. Pp. 267-281.

Alexander, M. P. A. 1980. Versatile stain for polen fungi, yeast and bacteria. Stain Technology 55(1): 13-18. DOI: https://doi. org/10.3109/10520298009067890

Anderson, L. C. 1972. Flaveria campestris (Asteraceae): A case of polyhaploidy or relic ancestral diploidy? Evolution 26(4): 671-673. DOI: https://doi.org/10.1111/j.1558-5646.1972. tb01974.x

Baker, H. G. 1955. Self-compatibility and establishment after long distance dispersal. Evolution 9(3): 347-349. DOI: https:// doi.org/10.2307/2405656

Bártoli, A., C. M. Baeza, N. D. Bayón, J. M. Bonifacino, G. Delucchi, M. Dematteis, S. E. Freire, D. Giuliano, D. Gutiérrez, L. Iharlegui, L. Katinas, A. A. Sáenz, G. Sancho, N. Soria, T. F. Stuessy, R. D. Tortosa, K. Tremetsberger y E. Uturbey. 2008. In: Zuloaga, F. O., O. Morrone y M. J. Belgrano (eds.). Catálogo de Plantas Vasculares del Cono Sur II. Monographs in Systematic Botany from the Missouri Botanical Garden. St. Louis, Missouri, USA. Pp. 985-1636.
Beuzenberg, E. J. y J. B. Hair. 1984. Contributions to a chromosome atlas of the New Zealand flora - 27: Compositae. New Zealand Journal of Botany 22(3): 353-356. DOI: https://doi. org/10.1080/0028825X.1984.10425266

Charleswort, D. y B. Charlesworth. 1987. Inbreeding depression and its evolutionary consequences. Annual Review of Ecology and Systematics 18: 237-268. DOI: https://doi. org/10.1146/annurev.es.18.110187.001321

Chai, M., F. Shi, K. S. He, R. Li, D. Cao y Z. He. 2012. Effects of saline-alkaline stress on early growth strategy and colonization success of Flaveria bidentis (I.) Kuntze (Asteracae) - a new exotic plant in northern China. Polish Journal of Ecology 60(3): 559-565.

Darlington, C. D. 1939. The evolution of genetic systems. Cambridge University Press. Cambridge, UK. 149 p.

Di Rienzo, J. A., F. Casanoves, M. G. Balzarini, L.González, M.Tablada y C. W. Robledo. 2012. Grupo InfoStat, Facultad Ciencias Agrarias. Universidad Nacional de Córdoba. Argentina. http://www.infostat.com.ar (consultado febrero de 2019).

Gabriele, M., A. I. Honfi, M. Gabriele, H. J. Debat y J. R. Daviña. 2009. Caracterización morfológica y cromosómica de Commelina benghalensis (Commelinaceae) de Argentina. Gayana Botánica 66(1): 18-27. DOI: https://dx.doi.org/10.4067/ S0717-66432009000100003

Hagerup, O. 1951. Pollination in the Faroes-In spite of rain and poverty in insects. Det Kongelige Danske Videnskabernes Selskab Biologiske Meddelelse 18(15): 1-48.

Heslop-Harrison, J. y Y. Heslop-Harrinson. 1970. Evaluation of pollen viability by enzymatically induced fluorescence; intracellular hydrolysis of fluorescein diacetate. Stain Technology 45(3): 115-120. DOI: https://doi. org/10.3109/10520297009085351

Hunziker, J. H., A. Escobar, C. C. Xifreda y J. C. Gamerro. 1990. Estudios cariológicos en Compositae. VI. Darwiniana 30(1-4): 115-121.

Keil, D. J. y R. F. Stuessy. 1977. Chromosome counts of Compositae from Mexico and the United States. American Journal of Botany 64(6): 791-798. DOI: https://doi. org/10.1002/j.1537-2197.1977.tb11921.x

Koella, J. C. 1993. Ecological correlates of chiasma frequency and recombination index of plants. Biological Journal of the Linnean Society 48(3): 227-238. DOI: https://doi.org/10.1006/ bijl.1993.1016 
Kulshreshtha, V. B. y P. K. Gupta. 1979. Cytogenetic studies in the genus Helianthus L. Cytologia 44(2): 325-334. DOI: https:// doi.org/10.1508/cytologia.44.325

Levan, A, K. Fredga, A. Sanberg. 1964. Nomenclature for centromeric position on chromosomes. Hereditas52(2): 201-220. DOI: https://doi.org/10.1111/j.1601-5223.1964.tb01953.x

Lewis, W. H. 1980. Polyploidy in angiosperms: Dicotyledons. In: Lewis, W. H. (ed.) Polyploidy, biological relevance. Plenum Press. New York, USA. Pp. 254. DOI: https://doi. org/10.1007/978-1-4613-3069-1_14

Novara, L. J. y E. M. Petenatti. 2012. Asteraceae Bercht. \& J. Presl (Compositae Giseke) Tribu Helenieae Benth. \& Hook. Flora del Valle de Lerma 6(8): 1-46.

Petenatti, E. M. y L. Ariza Espinar. 1997. Asteraceae, parte 6. Tribu VI. Helenieae. Flora Fanerogámica Argentina 45: 3-35.

Powell, A. M. 1978. Systematics of Flaveria (Flaveriinae-Asteraceae). Annals Missouri Botanical Garden 65(2): 590-636. DOI: https://doi.org/10.2307/2398862

Powell, A. M. y S. A. Powell. 1978. Chromosome numbers in Asteraceae. Madroño 25(3): 160-169.

Proctor, M. y P. Yeo. 1979. The pollination of flower. Williams Collins Sons \& Co. Ltd. London, UK. 418 pp.

Reeves, A. 2001. MicroMeasure: a new computer program for the collection and analysis of cytogenetic data. Genome 44(3): 439-443. DOI: https://dx.doi.org/10.1139/gen-44-3-439

Richards, A. 1986. Plant Breeding Systems. George Allen \& Unwin (Publishers) Ltd. London, UK. 529 pp.
Robinson, H., A. M. Powell, R. M. King y J. F. Weedin. 1981. Chromosome numbers in Compositae, XII: Heliantheae. Smithsonian Contributions to Botany 52: 1-28.

Romero-Zarco, C. 1986. A new method for estimating karyotype asymmetry. Taxon 35(3): 526-530. DOI: https://doi. org/10.2307/1221906

Schwarz, G. 1978. Estimating the dimension of a model. The Annals of Statistics 6(2):461-464. DOI: https://doi. org/10.1214/aos/1176344136

Sharma, A. K. y A. Sharma. 1965. Chromosome techniques, theory and practice. Butterworth \& Co. London, UK. 474 pp.

Shi Li, R., Y. Gao Ru, G. Lu Zhan, X. Zheng Yun y L. Niu Yu. 2006. The chromosome number and karyotype analysis of Flaveria bidentis. Acta Agrestia Sinica 14(4): 387-389.

Stebbins, G. L. 1957. Self-fertilization and population variability in the higher plants. The American Naturalist 91(861): 337354. DOI: https://doi.org/10.1086/281999

Stebbins, G. L. 1971. Chromosomal evolution in higher plants. Edward Arnold (Publishers) Ltd. London, UK. 214 pp.

Turner, B. L. 1971. Taxonomy Sartwellia (Compositae Helenieae). Sida Contributions to Botany 4: 265- 273.

Turner, B. L. 1975. Taxonomy of Haploesthes (Asteaceae, Senecioneae). Wrightia 5: 108-115.

Zuloaga, F. O. y O. Morrone. 1999. Catálogo de las plantas vasculares de la República Argentina II. Dicotyledoneae. Monographs in Systematic Botany from the Missouri Botanical Garden 74: 1-1269. 